\title{
Knockdown of TRAF4 expression suppresses osteosarcoma cell growth in vitro and in vivo
}

\author{
WEITAO YAO, XIN WANG, QIQING CAI, SONGTAO GAO, JIAQIANG WANG and PENG ZHANG \\ Department of Bone and Soft Tumor, Affiliated Cancer Hospital of Zhengzhou University, \\ Henan Cancer Hospital, Zhengzhou, Henan 45000, P.R. China
}

Received March 31, 2014; Accepted September 18, 2014

DOI: $10.3892 /$ ijmm.2014.1948

\begin{abstract}
Tumor necrosis factor (TNF) receptor-associated factor 4 (TRAF4) is an adapter molecule that is overexpressed in certain cancers. TRAF4 is overexpressed in osteosarcoma tissues and osteosarcoma cells. Using the technique of RNA interference, the expression of TRAF4 in the human osteosarcoma Saos-2 cell line was shown to be downregulated. The proliferation, cell cycle arrest and apoptosis ability of Saos-2 cells were examined, as was tumor development in a xenograft mouse model. The results showed that the TRAF4 knockdown exerts inhibitory effects on the proliferation ability of Saos-2 cells and tumor development in a xenograft mouse model. Simultaneously, it was found that TRAF4 knockdown led to cell cycle arrest in the $\mathrm{G}_{1}$ phase and promoted Saos- 2 cell apoptosis. Following TNF- $\alpha$ treatment, the expression of nuclear factor $\kappa \mathrm{B}$ was significantly reduced in the TRAF4-small interfering RNA group. These results indicate that TRAF4 regulated osteosarcoma cell growth in vitro and in vivo, and offers a candidate molecular target for osteosarcoma prevention and therapy.
\end{abstract}

\section{Introduction}

Osteosarcoma is an aggressive bone tumor characterized by osteoblastic differentiation and malignant osteoid production (1). It is the most common type of primary malignant bone tumor affecting children and adolescents, and the overall 5 -year survival rate of osteosarcoma patients is $68 \%$ (2). The underlying mechanisms in osteosarcoma carcinogenesis have been investigated in the past few decades, however, the progress has been slow and the survival rate of patients has reached a plateau $(3,4)$. The current treatment of osteosarcoma requires multidisciplinary therapy, incorporating surgery and systemic chemotherapy (5). However, the therapies all

Correspondence to: Dr Weitao Yao, Department of Bone and Soft Tumor, Affiliated Cancer Hospital of Zhengzhou University, Henan Cancer Hospital, 127 Dong Ming Road, Zhengzhou, Henan 45000, P.R. China

E-mail: weitao_yao@126.com

Key words: tumor necrosis factor receptor-associated factor 4 , osteosarcoma, RNA interference, apoptosis have different side-effect profiles and a poor prognosis. Thus, there is a requirement for an improved understanding of the pathogenesis of osteosarcoma, and the development of more effective therapeutic targets for this disease.

The tumor necrosis factor (TNF) receptor-associated factor (TRAF) family were originally discovered as signaling adaptors that couple the cytoplasmic regions of receptors of the TNF-R super-family (6). There are seven known members of the TRAF family (TRAF1 to 7) in mammals, and these play an important role in regulating cell survival, proliferation and stress responses (7). The distinctive feature of all the TRAF proteins is a C-terminal TRAF domain, which is composed of a C-terminal $\beta$-sandwich (TRAF-C) and an N-terminal coiled-coil region (TRAF-N) (8). Different members of the TRAF family mediate different signals. TRAF4 was the first member of the TRAF protein family found to be upregulated in human carcinomas. Unlike other canonical TRAFs, TRAF4 only interacts with limited TNFR-family members, including the p75 neurotrophin receptor, lichenoid tissue reaction and glucocorticoid-induced TNFR (GITR) (9), and was originally identified as a protein localized in the nucleus of breast carcinoma cells (10). TRAF4 has also been found in $43 \%$ of 623 human tumor samples from the prostate, ovary, lung and colon, among others (11). Therefore, TRAF4 protein overexpression is a common characteristic of numerous human cancers. In the latter study, TRAF4 mRNA was overexpressed in small cell lung carcinoma, lung adenocarcinoma, colon, ovary and prostate carcinomas (11). The amplification and overexpression of TRAF4 indicated that it is not only a marker of human carcinomas, but also a candidate oncogene. However, the role and mechanism of TRAF4 in osteosarcoma remains unclear.

In the present study, the aim was to examine $T R A F 4$ expression in osteosarcoma tissue and osteosarcoma cells by reverse transcription-quantitative polymerase chain reaction (RT-qPCR) and western blot analysis and to observe the biological function of Saos-2 cells following TRAF4 knockdown by the RNA interference technique. These data may provide information for prognosis prediction and targeted therapy for osteosarcoma.

\section{Materials and methods}

Specimens. Primary osteosarcoma tissues were obtained from biopsies in 38 patients prior to the administration of 
neo-adjuvant chemotherapy according to the Chinese National Ethical guidelines ('Code for Proper Secondary Use of Human Tissue', Chinese Federation of Medical Scientific Societies). In addition, adjacent normal bone tissue specimens were randomly obtained from 15 of these 38 osteosarcoma patients following surgical resection. The patients with osteosarcoma included $23(60.53 \%)$ males and $15(39.47 \%)$ females, aged 11-58 years, with a median age of 23 years. Without any preoperative treatment, all the 38 cases were pathologically diagnosed with osteosarcoma postoperatively. Informed consent was obtained from all the patients prior to entering the present study, and all the study protocols were approved by the Ethics Committee for Clinical Research of the Henan Cancer Hospital, (Henan, China).

Reagents. Mouse anti-TRAF4 antibody, mouse anti-B-cell lymphoma 2 (Bcl-2), mouse anti-Bax, mouse anti-nuclear factor $\kappa \mathrm{B}(\mathrm{NF}-\kappa \mathrm{B})$ were purchased from purchased from Santa Cruz Biotechnology, Inc. (Santa Cruz, CA, USA). The antibodies against $\beta$-actin were from Good HERE Biotech Inc. (Hangzhou, China). The horseradish peroxidase-conjugated goat anti-mouse secondary antibodies were obtained from Abgent Biotechnology Co., Ltd. (Suzhou, China).

Saos-2 cells culture. The human osteosarcoma cell line, sarcoma osteogenic (Saos-2), was purchased from the Shanghai Academy of Life Sciences (Shanghai, China). The cells were cultured in Dulbecco's modified Eagle's medium (Pierce, Rockford, IL, USA) and supplemented with $10 \%$ fetal bovine serum, $1 \%$ penicillin/streptomycin and $1 \%$ L-glutamine at $37^{\circ} \mathrm{C}$ under a humidified atmosphere of $5 \% \mathrm{CO}_{2}$.

TRAF4 knockdown by small interfering RNA (siRNA). Saos-2 cells were divided into three groups: Control (treated with Lipofectamine ${ }^{\circledR} 2000$ only), vector (treated with Lipofectamine 2000 and control siRNA), and TRAF4-siRNA group (treated with Lipofectamine 2000 and TRAF4 siRNA). The Saos-2 cells were seeded into 6-well plates and incubated overnight, and were subsequently transfected using Lipofectamine 2000 (Invitrogen, Carlsbad, CA, USA), according to the manufacturer's instructions, with slight modifications. The TRAF4 sense sequence was: 5'-CACCAGCACATTCGAAAGCGA-3' (GeneChem Co., Ltd., Shanghai, China) (12). For every $1 \times 10^{5}$ cells, $0.5 \mu \mathrm{g}$ TRAF4 siRNA or control siRNA was diluted and mixed with $3 \mu \mathrm{l}$ transfection reagent. After mixing and incubating $30 \mathrm{~min}$, the transfection mixture was added to the cells. After $6 \mathrm{~h}$, the medium was changed to growth medium (13).

Cell proliferation. The MTS assay was used to determine cell proliferation, as previously described with a few modifications (14). Approximately $1 \times 10^{4}$ Saos- 2 cells were seeded in each well of a 96-microwell plate. After incubation for $48 \mathrm{~h}$, Cell Titer $96^{\circledR}$ AQueous One Solution Reagent (Hitachi, Tokyo, Japan), which is composed of the novel tetrazolium compound MTS and an electron-coupling reagent, phenazine methosulfate (PES, a redox intermediary), was added to each well according to the manufacturer's instructions. After $3 \mathrm{~h}$ in culture, the cell viability was determined by measuring the absorbance at $490 \mathrm{~nm}$ using an ELISA microplate reader (Invitrogen).
In vivo tumor growth. Athymic nude mice (Vital River Laboratory Animal Technology Co., Ltd., Beijing, China) were divided into two groups $(n=3)$ and injected in the right flank with control or TRAF4-siRNA Saos- 2 cells $\left(3 \times 10^{6}\right)$. The (a) tumor diameter and the (b) shortest track were measured using a vernier caliper every five days. The tumor volume (in cubic millimeter) was calculated according to the formula $\mathrm{V}=\mathrm{ab}^{2} / 2$ (15). On day 20, the tumors were removed and weighed. All the studies were performed in compliance with the Guide for the Care and Use of Laboratory Animals of Henan Province, China.

Determination of cell cycle by flow cytometry. Cell cycle analyses were performed as previously described, with a few modifications (16). The Saos-2 cells were cultured in serum-free medium for $24 \mathrm{~h}$ to complete synchronization; subsequently, cells were cultured in complete medium for $24 \mathrm{~h}$. The Saos- 2 cells were digested by trypsin, washed in PBS, and fixed by $70 \%$ cold ethanol at $-20^{\circ} \mathrm{C}$. The next day, Saos- 2 cells were washed with citrate phosphate buffer, followed by PBS, before the Saos-2 cells were incubated with RNAse solution $(100 \mu \mathrm{g} / \mathrm{ml})$ for $30 \mathrm{~min}$ at $37^{\circ} \mathrm{C}$. Subsequently, the Saos-2 cells were incubated in propidium iodide (PI) solution $(100 \mu \mathrm{g} / \mathrm{ml}$ in PBS) at room temperature for $30 \mathrm{~min}$. The cell cycle was detected by flow cytometry (Invitrogen). The experiment was repeated three times.

Determination of cell apoptosis by flow cytometry. Cell apoptosis analyses were performed as previously described, with a few modifications (17). Saos-2 cells were detached by trypsinization and washed twice in PBS, centrifuged at $1000 \mathrm{x} \mathrm{g}$ for $5 \mathrm{~min}$ and resuspended in $195 \mu \mathrm{l}$ Annexin V-fluorescein isothiocyanate (FITC)-binding buffer. A volume of $5 \mu \mathrm{l}$ Annexin V-FITC was added and the solution was mixed. Subsequently, Saos-2 cells were stained in the dark for $10 \mathrm{~min}$ at room temperature. Following this, Saos-2 cells were centrifuged at $1000 \mathrm{x}$ g for $5 \mathrm{~min}$ and resuspended in $190 \mu \mathrm{l}$ Annexin V-FITC-binding buffer. Finally, $10 \mu \mathrm{l}$ propidium iodide-staining solution was added and mixed. Saos- 2 cells were maintained on ice in the dark and immediately subjected to flow cytometric analysis. The data were analyzed using the CellQuest software (BD Biosciences, San Jose, CA, USA). The experiment was repeated three times.

Reverse transcription-quantitative polymerase chain reaction ( $R T$-qPCR). The tissue sample was cryopreserved in liquid nitrogen. Total RNA was extracted from cultured cells and tissue samples using the TRIzol Reagent (Invitrogen) according to the manufacturer's instructions. RT-qPCR was performed with a SuperRT One-Step RT-PCR kit (Jiangsu Jiangnan Biotechnology Co., Ltd., Jiangsu, China) according to the manufacturer's instructions. The primer sequences used for RT-qPCR were as follows: TRAF-4 forward, 5'-CTGGCTAA ACCACAGCACGTC-3'; and reverse, 5'-TCGCTTTCGAAT GTCCTGG-3' (18). The $25 \mu 1$ reaction mixtures contained $12.5 \mu \mathrm{l} 2 \mathrm{X}$ One Step RT-qPCR buffer, $0.5 \mu \mathrm{M}$ reverse primer, $0.5 \mu \mathrm{M}$ forward primer, $0.9 \mu \mathrm{l}$ enzymix, $90 \mathrm{ng}$ RNA template and $0.5 \mu \mathrm{M}$ probe. PCR conditions for the reverse transcription used to obtain cDNA were as follows: $45^{\circ} \mathrm{C}$ for $10 \mathrm{~min}$, pre-denaturation at $95^{\circ} \mathrm{C}$ for $10 \mathrm{~min}$ and subsequently 45 cycles 

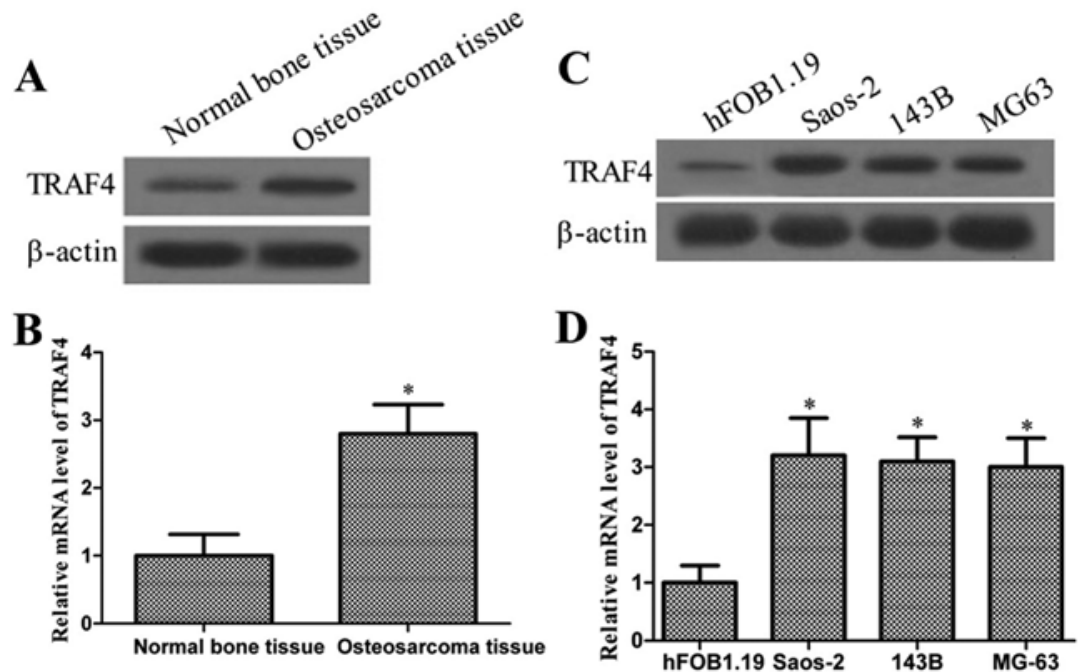

Figure 1. TRAF4 is overexpressed in osteosarcoma tissues and osteosarcoma cells. (A and C) Western blot analysis was performed to examine TRAF4 expression in osteosarcoma tissues, normal bone tissues, several osteosarcoma cell lines and normal human osteoblastic cells, hFOB1.19. $\beta$-actin was used as a loading control. (B and D) TRAF4 mRNA expression was determined in osteosarcoma tissues, normal bone tissues, several osteosarcoma cell lines and the normal human osteoblastic cells, hFOB1.19. Columns, means; bars, standard deviation $(\mathrm{n}=3)($ ( $\mathrm{P}<0.05$ vs. control). All the experiments were repeated at least three times.TRAF4, tumor necrosis factor receptor-associated factor.

at $95^{\circ} \mathrm{C}$ for $15 \mathrm{sec}$ and $60^{\circ} \mathrm{C}$ for $45 \mathrm{sec}$. This was performed using the ABI 7500 Real-Time PCR system (Applied Biosystems, Foster City, CA, USA). Relative quantification of gene expression was performed using the $2^{-\Delta \Delta C t}$ method and with $\beta$-actin mRNA as an internal control (19).

Western blot analysis. Western blot analyses were performed as previously described (20). Tissue sample or cells were homogenized in lysis buffer and centrifuged at $4^{\circ} \mathrm{C}$ for $30 \mathrm{~min}$ at $16,000 \mathrm{x} \mathrm{g}$. The supernatant was collected and the same amount of protein from each sample was separated by sodium SDS-PAGE on a $12 \%$ gel and transferred to a nitrocellulose membrane. The following anti-TRAF4 or anti- $\beta$-actin was used and, subsequently, horseradish peroxidase-conjugated secondary antibodies were added (Invitrogen). The proteins were briefly incubated with an enhanced chemiluminescence reagent (Millipore, Billerica, MA, USA) and visualized on X-ray film.

Statistical analysis. The SPSS 19.0 software (IBM Corp., Armonk, NY, USA) was used to analyze the associated data with a t-test. $\mathrm{P}<0.05$ was considered to indicate a statistically significant difference.

\section{Results}

TRAF4 mRNA and protein expression in osteosarcoma tissues and osteosarcoma cells. To verify the expression of TRAF4 in osteosarcoma, the protein and mRNA levels of TRAF4 in osteosarcoma tissue and in a human osteosarcoma cell line array were determined. As shown in Fig. 1A and B, the TRAF4 protein and mRNA expressions in osteosarcoma tissues were significantly higher compared to normal bone tissues $(\mathrm{P}<0.05)$. Consistent with observations from samples, the protein and mRNA expressions of TRAF4 were higher in the osteosarcoma cells, Saos-2, 143B and MG63, compared to the normal human osteoblastic cells, hFOB1.19 (Fig. 1C
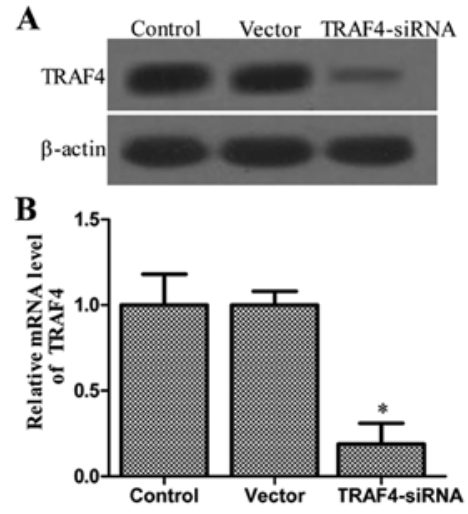

Figure 2. Expression of TRAF4 in Saos-2 cells. (A) Western blot analysis of TRAF4 expression $48 \mathrm{~h}$ after transfection with TRAF4-siRNA and control siRNA. $\beta$-actin was used as a loading control. (B) TRAF4 mRNA expression was detected by RT-qPCR $48 \mathrm{~h}$ after transfection with TRAF4-siRNA and control siRNA. All the experiments were repeated at least three times and all data are reported as mean \pm standard deviation $(n=3)\left({ }^{*} \mathrm{P}<0.05\right.$ vs. control). TRAF4, tumor necrosis factor receptor-associated factor; RT-qPCR, reverse transcription-quantitative polymerase chain reaction; siRNA, small interfering RNA.

and D). These results indicated that TRAF4 may be a critical molecule in osteosarcoma development.

Determination of transfection effects. On the basis of the observations, it was hypothesized that TRAF4 may affect the tumorigenic properties in osteosarcoma. Thus, the stable knockdown TRAF4 Saos-2 line was generated. To test the efficiency of TRAF4 transfection, western blotting and RT-qPCR were employed to determine the expression level of the protein and mRNA. As shown in Fig. 2, expression levels of the TRAF4 protein and mRNA were significantly decreased in the TRAF4 siRNA-transfected group. There was no significant difference in the expression level of TRAF4 protein and mRNA between the control and vector groups, which also demonstrated that 

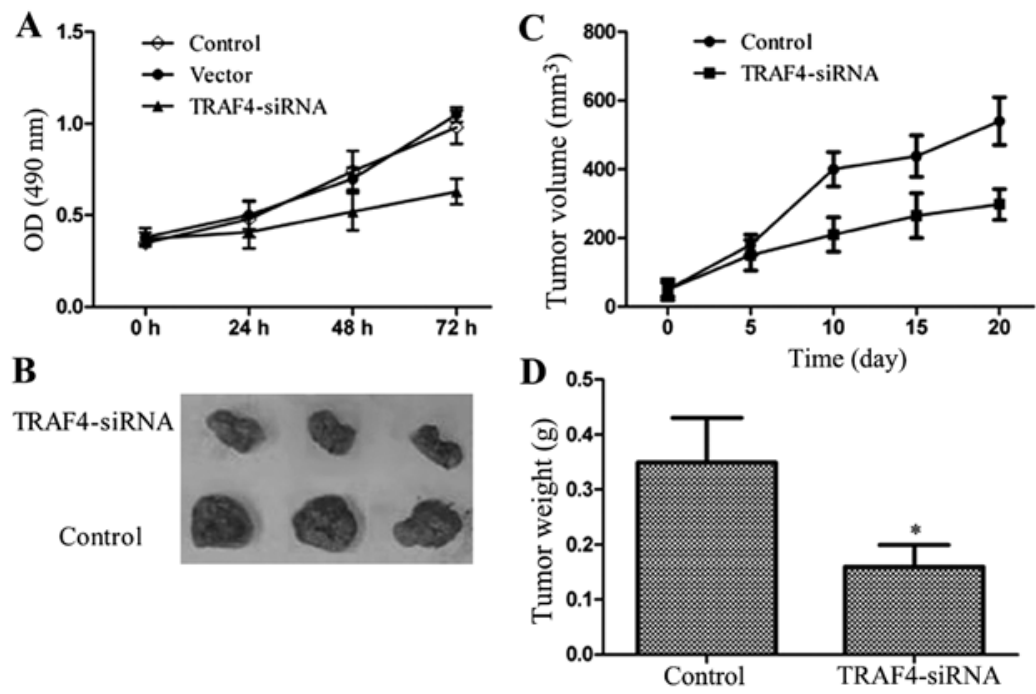

Figure 3. Effects of TRAF4 on Saos-2 cells in vitro and in vivo. (A) An MTS assay was conducted to assess the effect of TRAF4 on cell proliferation every $24 \mathrm{~h}$ after transfection, for up to $72 \mathrm{~h}$. All the experiments were repeated at least three times. All data are mean \pm standard deviation $(\mathrm{n}=3)\left({ }^{*} \mathrm{P}<0.05 \mathrm{vs}\right.$. control). (B) In vivo experiments. Tumors dissected from each group are shown as described in Materials and methods. (C) Tumor volume growth curve of Saos-2 grafts in mice. "Significant difference $(\mathrm{P}<0.05)$ in tumor volume $\left(\mathrm{mm}^{3}\right)$. (D) The tumor weights of the two groups. ${ }^{~} \mathrm{P}<0.05$ vs. control. TRAF4, tumor necrosis factor receptor-associated factor.

Lipofectamine and control siRNA did not affect the expression of TRAF4 protein and mRNA. Collectively, these data also demonstrated that the TRAF4 protein and mRNA were inhibited in Saos-2 cells.

Effects of TRAF4 on Saos-2 cells in vitro and in vivo. The impact of TRAF4 on Saos-2 cell proliferation was determined via an MTS assay every $24 \mathrm{~h}$ after transfection, for up to $72 \mathrm{~h}$. The results revealed that the viability of the cell was, to a certain extent, inhibited by TRAF4 in a time-dependent manner. As shown in Fig. 3, the TRAF4-transfected group grew more slowly compared to the control and vector groups. Furthermore, xenograft growth of Saos-2 cells in athymic nude mice was also attenuated following the knockdown of TRAF4 (Fig. 3B). These results demonstrated that the downregulation of TRAF4 inhibited the tumorigenic properties of Saos-2 cells.

Effects of TRAF4 on cell cycle progression in Saos-2 cells. Flow cytometry cell cycle analysis showed that the Saos- 2 cells in the TRAF4-siRNA group had significantly more cells in the $\mathrm{G}_{1}$ phase and significantly fewer cells in the $S$ and $G_{2}$ phases compared to the control and vector groups $(\mathrm{P}<0.05)$ (Fig. 4). These results indicated that the downregulation of TRAF4 may affect the cell cycle distribution of Saos-2 cells.

Effects of TRAF4 on apoptosis in Saos-2 cells. Apoptosis of Saos-2 cells was detected via PI staining and the Annexin V method after $48 \mathrm{~h}$ of TRAF4 transfection, followed by flow cytometry. As shown in Fig. 5A, there was an extremely low level (10.6 and 10.5\%) of Saos-2 cell apoptosis in the control and vector groups, but the percentage of apoptosis significantly increased to $27.3 \%(\mathrm{P}<0.05)$ in the TRAF4-siRNA group. Furthermore, the percentage of apoptotic cells did not differ significantly between the control and vector groups $(\mathrm{P}<0.05)$.

To investigate whether TRAF4 induces apoptosis in Saos-2 cells, the possible molecular mechanisms of TRAF4 associated with apoptosis were investigated. Thus, the expression of Bcl-2 and Bax proteins were measured in Saos-2 cells (Fig. 5C). The results showed that the expression of Bcl-2 decreased and the expression of Bax was simultaneously upregulated in the TRAF4-siRNA group compared to the control and vector groups $(\mathrm{P}<0.05)$. The apoptosis rate was significantly higher due to the upregulation of pro-apoptotic genes. These data revealed that TRAF4 plays a critical role in promoting apoptosis of Saos-2 cells.

Effect of TRAF4 on NF- $\kappa B$ expression. TRAF4 is an important regulatory factor for the expression of NF- $\mathrm{KB}(28)$. The expression of NF- $\mathrm{kB}$ was examined in the Sao- 2 cell line by western blotting. Compared to the control and vector groups, the TRAF4-siRNA group had significantly reduced expression levels of NF- $\kappa$ B following TNF- $\alpha$ treatment (Fig. 6). These results indicate that TRAF4 may promote the activation of NF- $\mathrm{KB}$ induced by TNF- $\alpha$ in Saos-2 cells.

\section{Discussion}

Osteosarcoma is the most common primary malignant bone lesion and a highly malignant tumor with extensively destructive potential (21). Despite great advancements in the diagnosis and treatment of osteosarcoma thus far, substantial improvements in overall survival rate have been elusive and overall survival has remained relatively constant for over 2 decades (22). Thus, establishing the molecular mechanism of tumorigenesis and the progression of osteosarcoma and exploring the effective treatments for osteosarcoma is vital.

TRAF4 was initially identified as an overexpressed gene in human breast carcinoma (23). Overexpression of the TRAF4 protein is the consequence of its gene amplification in approximately one-quarter of human carcinomas (11). Several previous studies have hypothesized that TRAF4 may be involved in apoptosis. For instance, TRAF4 provides 

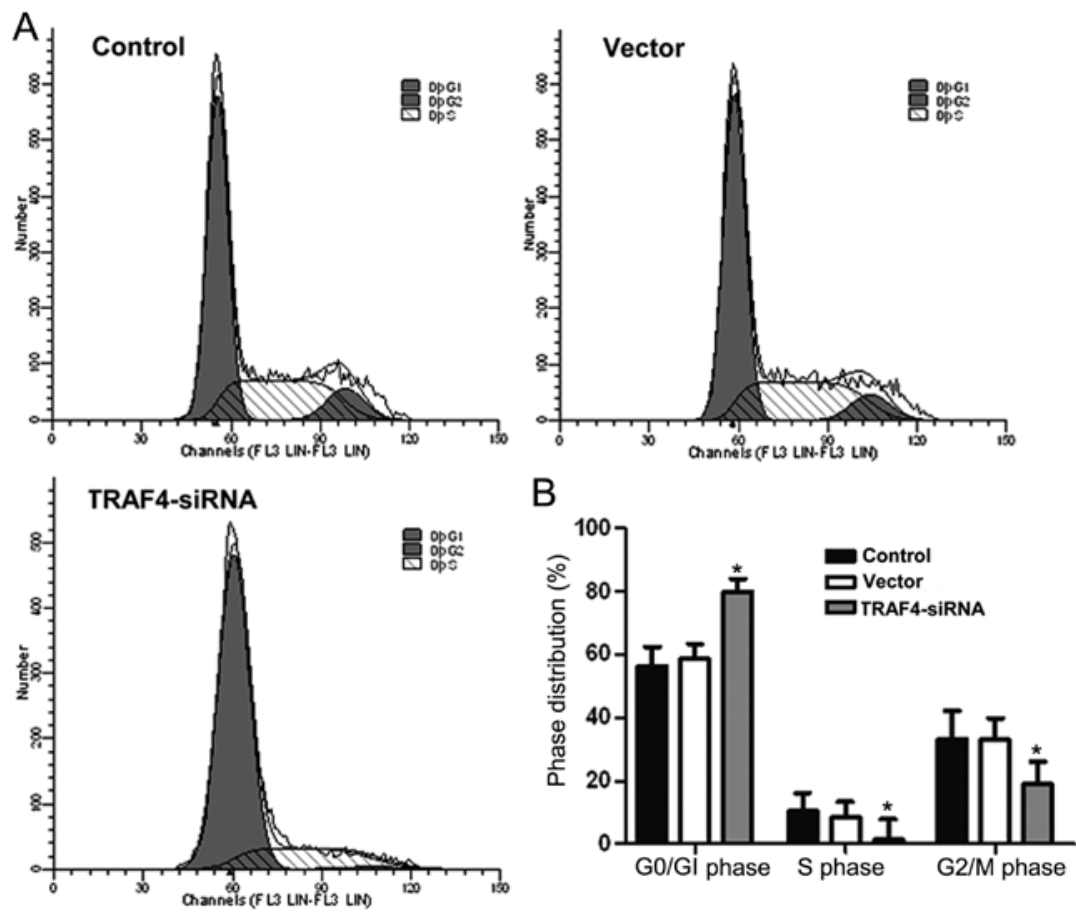

Figure 4. Effects of TRAF4 on cell cycle progression in Saos-2 cells. (A) Cell cycle progression was evaluated by flow cytometry after $48 \mathrm{~h}$ of TRAF4 transfection. (B) Percentages of cell cycle distribution among different groups are presented as a histogram graph. The experiments were repeated three times and the data are shown as mean \pm standard deviation. ${ }^{*} \mathrm{P}<0.05$ vs. control. TRAF4, tumor necrosis factor receptor-associated factor.
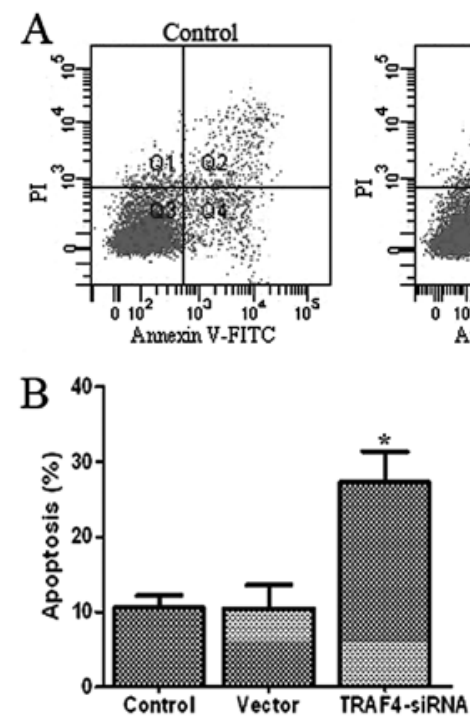
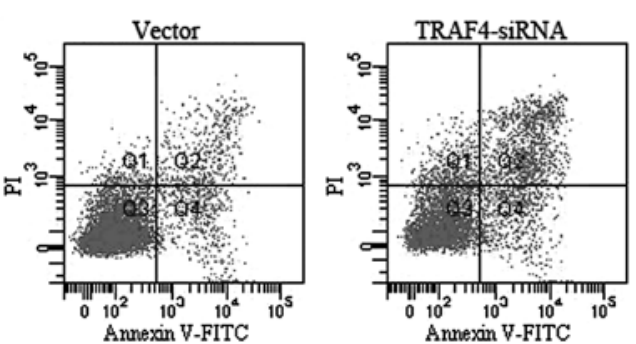

$\mathrm{C}$

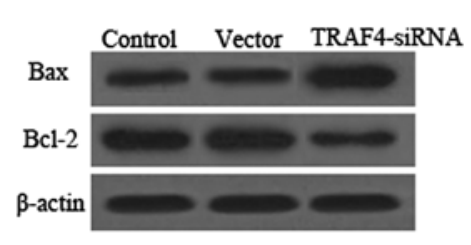

Figure 5. Effects of TRAF4 on Saos-2 cell apoptosis. (A) Saos-2 cell apoptosis was detected via PI staining and the Annexin V method after $48 \mathrm{~h}$ of TRAF4 transfection, followed by flow cytometry. (B) Columns show the mean of data obtained from three independent experiments. Bar is the standard deviation. ${ }^{*} \mathrm{P}<0.05$ vs. control. (C) TRAF4 modulates the expression of Bcl-2 and Bax in Saos-2 cells. The expression levels of Bcl-2 and Bax were determined by western blot analysis. All the experiments were repeated at least three times. TRAF4, tumor necrosis factor receptor-associated factor; PI, propidium iodide; Bcl-2, B-cell lymphoma 2; FITC, fluorescein isothiocyanate; siRNA, small interfering RNA.

resistance to an apoptotic stimulus in HEK293 cells (24). By contrast, Sax and El-Deiry (25) reported that TRAF4 may play a role in p53-mediated pro-apoptotic signaling in response to cellular stress. However, depending on the study the role of TRAF4 in apoptosis is controversial. Currently, TRAF4 has been found highly expressed in human carcinomas, but its biological functions in tumorigenesis remain unclear.

The results of the present study showed that TRAF4 is overexpressed in osteosarcoma tissues and cell lines. To study

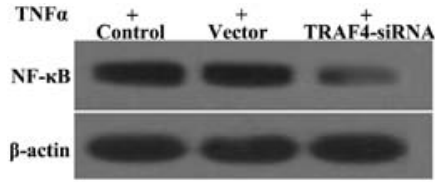

Figure 6. Effect of TRAF4 on NF-кB expression. The protein level of NF-кB was determined by western blot analysis, $\beta$-actin was used as a loading control. All the experiments were repeated at least three times. TRAF4, tumor necrosis factor (TNF) receptor-associated factor; NF- $\kappa B$, nuclear factor $\kappa \mathrm{B}$; siRNA, small interfering RNA. 
the function of TRAF4, a stable TRAF4 knockdown Saos-2 cell line was generated and the effects of the downregulation of TRAF4 on the proliferation, cell cycle arrest and apoptosis ability in the Saos- 2 cell line, as well as tumor development in a xenograft mouse model were examined. The study showed that the knockdown of TRAF4 inhibited the proliferation of the Saos-2 cell line in vitro and slowed down tumor growth in a xenograft mouse model. These results indicated that TRAF4 plays a crucial role in osteosarcoma carcinogenesis. However, TRAF4 as a p53-regulated pro-apoptotic gene in a p53 temperature-sensitive cell line VM10 induced apoptosis and inhibited colony formation (25). Simultaneously, TRAF4 knockdown may lead to cell cycle arrest in the $G_{1}$ phase and promote Saos-2 cell apoptosis. Knockdown of TRAF4 can downregulate $\mathrm{Bcl}-2$ expression and upregulate Bax expression. TRAF4 knockdown possibly induces Saos-2 cell apoptosis by inhibiting Bcl-2 and activating Bax expression. This apparent discrepancy indicates that the same gene can perform various biological functions that are dependent upon the form of the cell and the type of stimulation.

Previous studies have indicated that TRAF4 positively regulates GITR-induced $\mathrm{NF}-\kappa \mathrm{B}$ activation (26). NF- $\kappa \mathrm{B}$ plays an essential role in preventing TNF- $\alpha$-induced cell death (27). Therefore, the expression of $\mathrm{NF}-\kappa \mathrm{B}$ was examined. Following TNF- $\alpha$ treatment, NF- $\kappa$ B exhibited downregulation in Saos-2 cells from the TRAF4-siRNA group compared to the control and vector groups. These data indicate that the downregulation of TRAF4 may be associated with the downregulation of $N F-\kappa B$, and that the downregulation of $N F-\kappa B$ may be further responsible for the induced apoptotic ability. However, its exact mechanism requires further research.

These results indicate that the knockdown of TRAF4 may play an important role in carcinogenesis and the development of osteosarcoma. Additional studies are required to investigate the specific mechanisms underlying the effects of TRAF4 in the tumorigenesis, xenograft tumor growth, cell cycle arrest and apoptosis of osteosarcoma. These results suggest that TRAF4 is a good molecular target for the prevention and treatment of osteosarcoma.

\section{References}

1. Zhao H, Li M, Li L, Yang X, Lan G and Zhang Y: MiR-133b is down-regulated in human osteosarcoma and inhibits osteosarcoma cells proliferation, migration and invasion, and promotes apoptosis. PLoS One 8: e83571, 2013.

2. Shimbo K, Miyaki S, Ishitobi H, Kato Y, Kubo T, Shimose $S$ and Ochi M: Exosome-formed synthetic microRNA-143 is transferred to osteosarcoma cells and inhibits their migration. Biochem Biophys Res Commun 445: 381-387, 2014.

3. Pierz KA, Womer RB and Dormans JP: Pediatric bone tumors: osteosarcoma ewing's sarcoma, and chondrosarcoma associated with multiple hereditary osteochondromatosis. J Pediatr Orthop 21: 412-418, 2001

4. Yang C, Hou C, Zhang H, Wang D, Ma Y, Zhang Y, Xu X, Bi Z and Geng S: miR-126 functions as a tumor suppressor in osteosarcoma by targeting Sox2. Int J Mol Sci 15: 423-437, 2014.

5. Xie ZG, Xie Y and Dong QR: Inhibition of the mammalian target of rapamycin leads to autophagy activation and cell death of MG63 osteosarcoma cells. Oncol Lett 6: 1465-1469, 2013.
6. Xie P: TRAF molecules in cell signaling and in human diseases. J Mol Signal 8: 7, 2013.

7. Wajant $H$, Henkler $F$ and Scheurich P: The TNF-receptor-associated factor family: scaffold molecules for cytokine receptors, kinases and their regulators. Cell Signal 13: 389-400, 2001

8. Ha H, Han D and Choi Y: TRAF-mediated TNFR-family signaling. Curr Protoc Immunol Chapter 11: Unit 11.9D, 2009.

9. Arron JR, Walsh MC and Choi Y: TRAF-mediated TNFR-family signaling. Curr Protoc Immunol Chapter 11: Unit 11.9D, 2002.

10. Tomasetto C, Regnier $\mathrm{CH}$ and Rio MC: TRAF-4 expression in breast carcinomas. Am J Pathol 153: 2007-2008, 1998.

11. Camilleri-Broët S, Cremer I, Marmey B, Comperat E, Viguié F, Audouin J, Rio MC, Fridman WH, Sautès-Fridman C and Régnier CH: TRAF4 overexpression is a common characteristic of human carcinomas. Oncogene 26: 142-147, 2007.

12. Marinis JM, Homer CR, McDonald C and Abbott DW: A novel motif in the Crohn's disease susceptibility protein, NOD2, allows TRAF4 to down-regulate innate immune responses. J Biol Chem 286: 1938-1950, 2011.

13. Qin GQ, He HC, Han ZD, Liang YX, Yang SB, Huang YQ, Zhou L, Fu H, Li JX, Jiang FN and Zhong WD: Combined overexpression of HIVEP3 and SOX9 predicts unfavorable biochemical recurrence-free survival in patients with prostate cancer. Onco Targets Ther 7: 137-146, 2014.

14. Tayarani-Najaran Z, Asili J, Aioubi E and Emami SA: Growth inhibition and apoptosis induction of Salvia chloroleuca on MCF-7 breast cancer cell line. Iran J Pharm Res 12: 789-799, 2013.

15. Zhang B, Lu Z, Hou Y, Hu J and Wang C: The effects of STAT3 and Survivin silencing on the growth of human bladder carcinoma cells. Tumour Biol 35: 5401-5407, 2014.

16. Meng Q, Zheng M, Liu H, Song C, Zhang W, Yan J, Qin L and Liu X: TRAF6 regulates proliferation, apoptosis, and invasion of osteosarcoma cell. Mol Cell Biochem 371: 177-186, 2012.

17. Li X, Huang T, Jiang G, Gong W, Qian H and Zou C: Synergistic apoptotic effect of crocin and cisplatin on osteosarcoma cells via caspase induced apoptosis. Toxicol Lett 221: 197-204, 2013.

18. Guo F, Sun A, Wang W, He J, Hou J, Zhou P and Chen Z: TRAF1 is involved in the classical NF- $\kappa \mathrm{B}$ activation and CD30-induced alternative activity in Hodgkin's lymphoma cells. Mol Immunol 46: 2441-2448, 2009.

19. Zheng H, Liu C, Ou Y, Zhang Y and Fu X: Total saponins of Panax notoginseng enhance VEGF and relative receptors signals and promote angiogenesis derived from rat bone marrow mesenchymal stem cells. J Ethnopharmacol 147: 595-602, 2013.

20. Yang WT and Zheng PS: Promoter hypermethylation of KLF4 inactivates its tumor suppressor function in cervical carcinogenesis. PLoS One 9: e88827, 2014.

21. Garrington GE, Scofield HH, Cornyn J and Hooker SP: Osteosarcoma of the jaws. Analysis of 56 cases. Cancer 20: 377-391, 1967.

22. Geller DS and Gorlick R: Osteosarcoma: a review of diagnosis, management, and treatment strategies. Clin Adv Hematol Oncol 8: 705-718, 2010.

23. Tomasetto C, Régnier C, Moog-Lutz C, Mattei MG, Chenard MP, Lidereau R, Basset P and Rio MC: Identification of four novel human genes amplified and overexpressed in breast carcinoma and localized to the q11-q21.3 region of chromosome 17 . Genomics 28: 367-376, 1995.

24. Fleckenstein DS, Dirks WG, Drexler HG and Quentmeier H: Tumor necrosis factor receptor-associated factor (TRAF) 4 is a new binding partner for the p70S6 serine/threonine kinase. Leuk Res 27: 687-694, 2003.

25. Sax JK and El-Deiry WS: Identification and characterization of the cytoplasmic protein TRAF4 as a p53-regulated proapoptotic gene. J Biol Chem 278: 36435-36444, 2003.

26. Esparza EM and Arch RH: TRAF4 functions as an intermediate of GITR-induced NF- $\kappa$ B activation. Cell Mol Life Sci 61: 3087-3092, 2004.

27. Beg AA and Baltimore D: An essential role for NF-kappaB in preventing TNF-alpha-induced cell death. Science 274: 782-784, 1996. 